\title{
Mean Pressure Gradient
}

National Cancer Institute

\section{Source}

National Cancer Institute. Mean Pressure Gradient. NCI Thesaurus. Code C147152.

The arithmetic average of the quantitative measurement of the pressure gradient across a predetermined point. 